\title{
Comportamiento de la precipitación y cuantificación del balance hídrico en la cuenca alta del río Atrato en el departamento del Chocó
}

\section{Behavior of precipitation and quantification of the water balance of the river Atrato high basin in the department of Chocó}

\author{
William Murillo López ${ }^{1,2}$, Samir Córdoba Machado ${ }^{1,2}$, Reiner Palomino Lemus $^{1,2}$
}

\section{RESUMEN}

Se presenta el comportamiento de la precipitación media a través de dos métodos, media aritmética normal y la basada en el análisis de isoyetas; también se realiza una cuantificación del balance hídrico en la cuenca parte

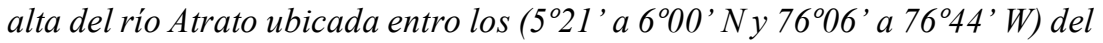
departamento del Chocó, empleándose para ello los registros mensuales (promedios mensuales) registrados durante 20 años (1983 a 2003). Se encontró que el comportamiento de la precipitación en la cuenca alta del río Atrato es de tipo bimodal, presentando dos valores máximos y dos valores mínimos. El primer máximo hacia los meses de abril y mayo, y el segundo máximo entre los meses de julio y agosto. Estas son las temporadas donde la cuenca recibe la mayor cantidad de lluvia. La estación de Tutunendo fue la que presentó los mayores valores de precipitación seguido de la estación AP Quibdó, y las menores en El Carmen de Atrato. El estudio concluye con un cálculo del balance hídrico para la cuenca alta del río Atrato, donde puede apreciarse la gran contribución de las lluvias a la conformación de una escorrentía de gran consideración en la región.

Palabras clave: Precipitación media; Balance hídrico; Río Atrato; Precipitación media por isoyetas; Curva de intensidad, duración; Características de la cuenca alta del río Atrato.

\section{ABSTRAT}

This paper presents the behavior of average rainfall, through two methods, the normal mean arithmetic and the one based on an analysis of isoyetas. There is also a quantification of water balance in the upper basin located in the Atrato River $\left(5^{\circ} 21^{\prime}\right.$ to $6^{\circ} 00^{\prime} N$ and $76^{\circ} 06^{\prime}$ to $76^{\circ} 44^{\prime} W$ ) of the Department of Choco, using the monthly records (monthly averages) recorded during a period of 20 years (1983 to 2003). We find that the behavior of high precipitation in the watershed of the Atrato River is a bimodal, presenting two maxima and two thresholds. The first maximum towards the months of April and May, and the second highest among the months of July and August. These are the seasons in which the basin receives the largest amount of rainfall. The station was Tutunendo which presented the highest values of rainfall followed by Station AP Quibdo, and the lowest in El Carmen de Atrato. The study concludes with an estimate of water balance for the Atrato River high basin, in which the great contribution of rainfall to the formation of a runoff great consideration in the region can be seen.

Keywords: Average rainfall; Water balance; Atrato River; Average
1. Grupo de Investigación en Energía Solar y Meteorología, Universidad Tecnológica del Chocó, Quibdó, Colombia.

2. Docente Investigador, Departamento de Matemáticas y Física, Universidad Tecnológica del Chocó, Quibdó, Colombia. e-mail:wmlopez@hotmail.com e-mail:samostv@hotmail.com e-mail:cmaxtor@gmail.com

Recibido: agosto 6, 2008 Aceptado: agosto 29, 2008 


\section{Bioetnia Volumen 5 No 2 (julio-diciembre), 2008}

precipitation isoyetas; Curve intensity, duration; Characteristics of the upper basin River Atrato scope of meteorology and climatology.

\section{INTRODUCCIÓN}

La creciente necesidad de un conocimiento de la hidrología colombiana en áreas como el planeamiento territorial, la evaluación del potencial de aprovechamiento hidráulico, la planeación agrícola, entre otros, ha motivado el estudio de la distribución espacial sobre Colombia del comportamiento de largo plazo de las variables hidrológicas de mayor importancia (precipitación, evaporación, evapotranspiración, escorrentía). Hoy en día el departamento del Chocó ubicado en la región Pacífico de Colombia representa una de las fuentes hídricas más importantes del mundo, gracias a la gran cantidad de lluvias que se genera en la zona. La precipitación en el departamento del Chocó puede alcanzar fácilmente los 8.000 y 12.000 milímetros al año, ubicándola como una de las regiones más lluviosas del globo. Como consecuencia de las altas precipitaciones, el departamento del Chocó presenta una gran cantidad de ríos (potencial hídrico), de los cuales la mayoría se caracterizan por sus tramos relativamente cortos y alto caudal (Ramírez y Bustos, 1977). Además la región del Chocó es epitome de la selva húmeda tropical desde el punto de vista físico natural (Gentry, A., 1990, 40), de acuerdo con la terminología de las biozonas de Holdridge, es el único lugar del neotrópico en donde se encuentra el verdadero «bosque pluvial tropical».

Debido al potencial hídrico presente en el departamento se hace necesario estudios que permitan el desarrollo y aprovechamiento de estas fuentes naturales. Además en la región son poco los trabajos o investigaciones sobre balances hídricos o comportamiento precipitación media en una cuenca determinada, etc. En vista de lo anterior resulta conveniente la elaboración del estudio sobre el balance hídrico y el comportamiento de la precipitación media en la cuenca alta del río Atrato en el departamento del Chocó, porque permite generar conocimiento sobre este tipo de temas en la región y esgrime como metodología o guía para próximas investigaciones.

Antecedentes históricos. Según(Trojer, 1958) el Pacífico colombiano, está localizado dentro de la región de bajas presiones atmosféricas conocida como «concavidad ecuatorial» en donde convergen los vientos Alisios de cada hemisferio formando la ZCIT (zona de confluencia intertropical). En esta zona, el choque de masas de aire ascendentes con diferencias térmicas y gradientes de humedad, ocasionan la formación de una banda nubosa por procesos de convección. Debido a esto y a las condiciones físicas-geográficas (forma, orientación, rango de altitudes, topografía, vegetación, presencia tierra-agua, no continentalidad, distancia al litoral, etc.) que le son propias, el clima en la región del Pacífico colombiano se caracteriza por sus vientos variables y débiles (zona de calmas ecuatoriales), por su alta pluviosidad, abundante nubosidad y mucha humedad. Se cree que a causa de este tipo de circulación atmosférica (interacción tierra-atmósfera) que impera en la zona, es probable que en alguna parte de su geografía se encuentre el punto más húmedo del planeta.

Eslavas 1994, con el fin de realizar una clasificación climática en la región del Pacifico colombiano aplicando los modelos expuestos por Calda-Lang, Emmanuel De Martonne, C.W. Thornthwaite y W. Kôpen, elabora un balance hídrico para algunas estaciones climatológicas de la región, calculando de manera muy general datos de evapotranspiración, evaporación, precipitación media, etc.

En el año 2004 Donald Gabriels, Francisco Ovalles et al., con la ayuda de instituciones como PHI(Programa Hidrológico Internacional) la UNESCO y CAZALAC (Centro del Agua paraZonas Áridas y Semiáridas de América Latina y El Caribe) Elaboran la Guía metodológica para la elaboración del mapa de zonas áridas, semiáridas y subhúmedas secas de América Latina y El Caribe, en la que presentan algunas opciones para el cálculo de la evapotranspiración de referencia $\left(\mathrm{ET}_{0}\right)$, cuando no se dispone de la información para realizarlo con el protocolo de FAO/Penman-Monteith.

E1Servicio Nacional de Estudios Territoriales (SNET) y el Servicio Hidrológico Nacional del Salvador, realizan un balance hídrico para la Cuenca del Lago ILOPANGO, en el analizan el comportamiento de la precipitación media, la evapotranspiración, la evaporación media, el escurrimiento superficial entre otros. Vale decir que los trabajos expuestos fueron de gran ayuda para llevar a cabo el desarrollo del presente estudio, sin embargo, siguen siendo muy generales, lo que creó la necesidad de profundizar más en el tema.

\section{METODOLOGÍA}

Para el desarrollo del presente estudio, se plantearon varias etapas en un proceso que partió de la delimitación de la cuenca escogida (parte alta del rio Atrato), con la ayuda de cartografía 1:500.000, y la consecución de los datos climatológicos existentes en la zona de estudio. El detalle de la metodología en cada una de las etapas del proceso es el siguiente:

Debido al deficiente número de estaciones en la cuenca alta del río Atrato, se estimaron dos puntos de estaciones por interpolación, teniendo en cuenta la distribución de la precipitación por isoyetas, la altitud y el régimen de precipitaciones de estaciones cercanas al punto de estimación las cuales fueron estación Cerro de San Nicolás y Estación Bagadó, siendo la estación de Bagadó considerada como estación patrón.

Unidad de análisis. En este trabajo la unidad de análisis 


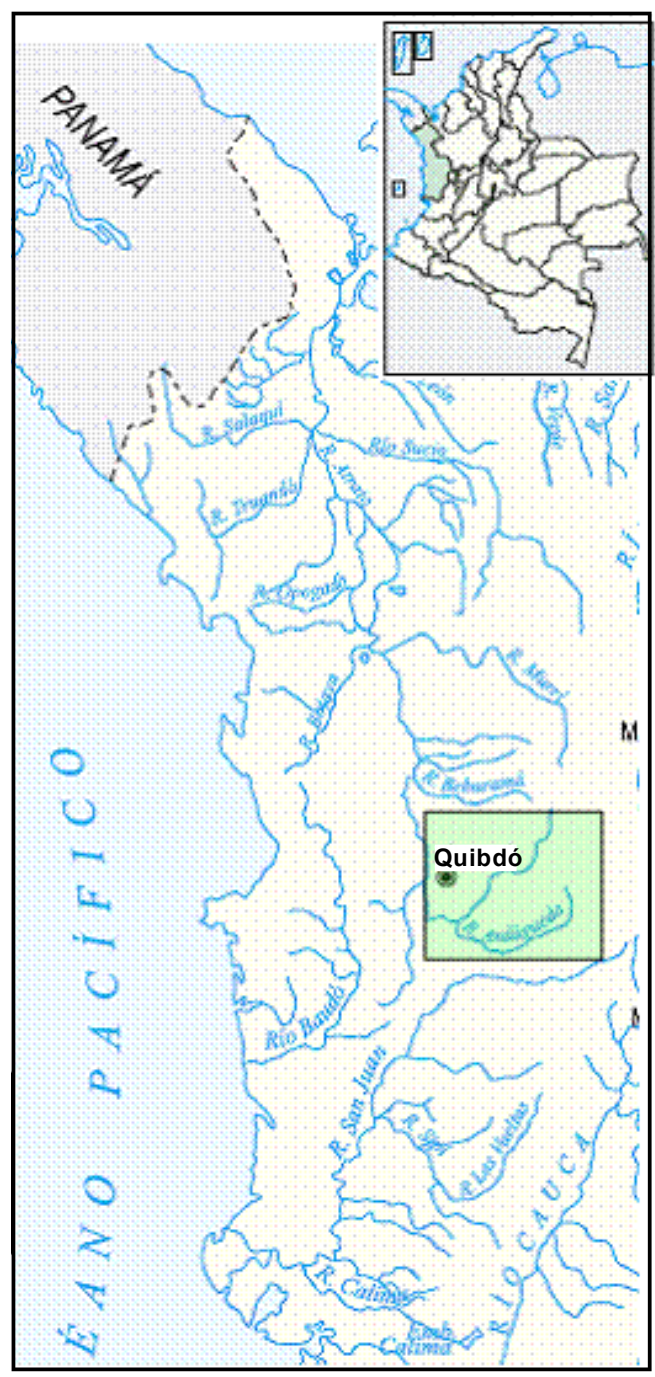

Figura 1. Departamento del Chocó Colombia, río Atrato (recuadro). Mapa digital IGAC

es la cuenca alta del río Atrato ubicada en el departamento del Chocó, Colombia (Figura 1). En primera medida, la cuenca es un elemento que no sólo presenta unas características climáticas determinadas, si no que integra otros elementos del ambiente como el agua, la vegetación y los suelos. En este sentido se planteó la elaboración del balance hídrico y el análisis del comportamiento de la precipitación en la cuenca alta del río Atrato.

Delimitación dela cuenca. Para realizar la delimitación de la cuenca alta del río Atrato, se planeó hacer el análisis detallado en mapas cartográficos a escala 1:1000.000 y 1:250.000; no obstante, ante la falta de esta información básica, debidamente estructurada para la zona, no fue posible hacer uso de ella. Se optó entonces por trabajar con cartografía a escala 1:500.000. Esta información básica comprende las curvas de nivel, las zonas y sub-zonas hidrográficas y divi-

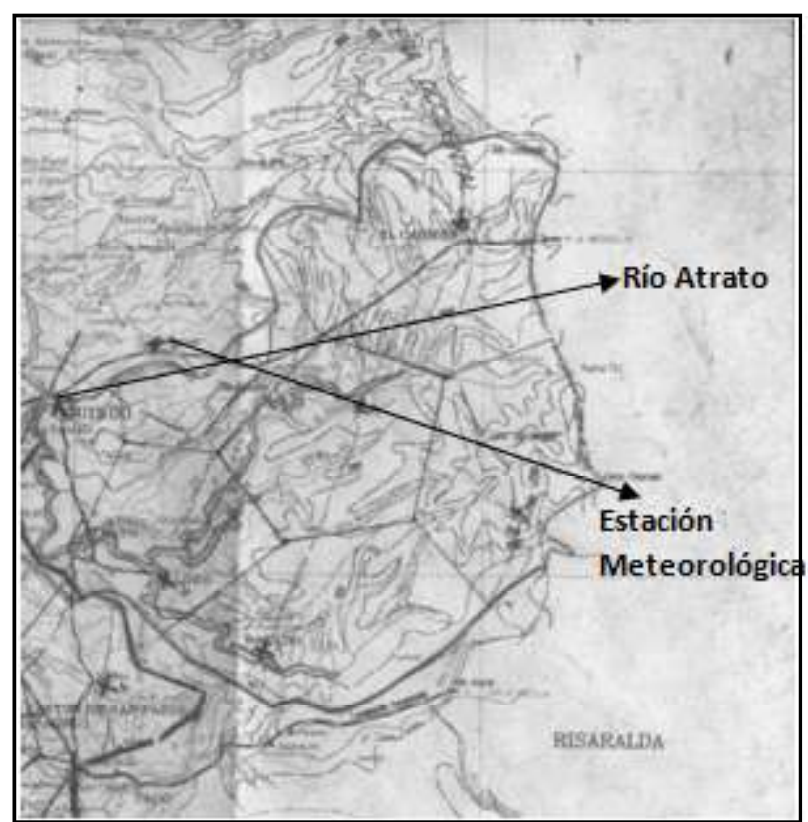

Figura 2. Demarcación del área de la cuenca alta del río Atrato en el departamento del Chocó, Colombia y ubicación de las estaciones meteorológicas existentes en su alrededor (cruces). Cartografía 1:500.000, IGAC.

sión político administrativa delárea de estudio. Adicionalmente se utilizó cartografía digital obtenida de la página web http:/ /webigac1.igac.gov. co/mapas.htm del IGAC (Instituto Geográfico Agustín Codazzi). Empleando la información anterior se hizo la demarcación de la cuenca, teniendo en consideración las línea divisoria de aguas, las curvas de nivel, los afluentes y ramificaciones del río Atrato, obteniendo un área para la cuenca entre $5^{\circ} 21^{\prime}$ a $6^{\circ} 00^{\prime}$ 'de latitud y longitud $76^{\circ} 06^{\prime}$ a 76⒋ $44^{\prime}$ (Figura 2).

Datos e información. Inicialmente se obtuvieron datos mensuales de precipitación en milímetro $(\mathrm{mm})$ de 10 estaciones meteorológicas alrededor de la cuenca alta del río Atrato, pertenecientes al Instituto de Hidrología, Meteorología y Estudios Ambientales de Colombia (IDEAM), de las cuales sólo 5 (Figura 2, cruces cumplían con un período de registro continuo, confiable y aceptable para el desarrollo del trabajo, a las cuales se le agregaron dos (Bagado y San Nicolás), como resultado de interpolaciones y métodos estadísticos, teniendo en cuentas estaciones cercanas (Tabla 1).

Por la escasez de datos de temperatura del aire para la región, se optó porutilizar los datos globales del proyecto Reanalis y National Center for Environmental Prediction/National Center for Atmospheric Research (NCEP/NCAR)(Kalnayetal., 1996), que presenta información de series conti-nuas de temperatura del aire desde 1967-2001, en diferentes niveles isobáricos incluyendo superficie, para el área de estudio. 
Tabla 1

Estaciones meteorológicas (IDEAM). Período de registro utilizado 1983-2003

\begin{tabular}{|c|c|c|c|c|}
\hline Nombre de la estación & Latitud $(\mathrm{N})$ & Longitud (W) & Elevación (msn) & Período utilizado \\
\hline Bagadó & $5043^{\prime}$ & $76^{\circ} 42^{\prime}$ & 500 & $1983-2003$ \\
\hline Lloró & $5^{0} 51^{\prime}$ & $76^{\circ} 52^{\prime}$ & 90 & 1983- 2003 \\
\hline San Nicolás & $5^{\circ} 59^{\prime}$ & $\begin{array}{ll}76^{\circ} & 13^{\prime}\end{array}$ & 2350 & 1983- 2003 \\
\hline El Piñol & $5^{\circ} 70^{\prime}$ & $76^{\circ} 31^{\prime}$ & 715 & $1983-2003$ \\
\hline Ap Quibdó & $5^{\circ} 70^{\prime}$ & $76^{\circ} 64^{\prime}$ & 53 & 1983- 2003 \\
\hline Tutunendo & $5^{\circ} 77^{\prime}$ & $76^{\circ} 53^{\prime}$ & 54 & 1983- 2003 \\
\hline Carmen de Atrato & $5^{\circ} 90^{\prime}$ & $\begin{array}{ll}76^{\circ} & 19^{\prime}\end{array}$ & 1850 & $1983-2003$ \\
\hline
\end{tabular}

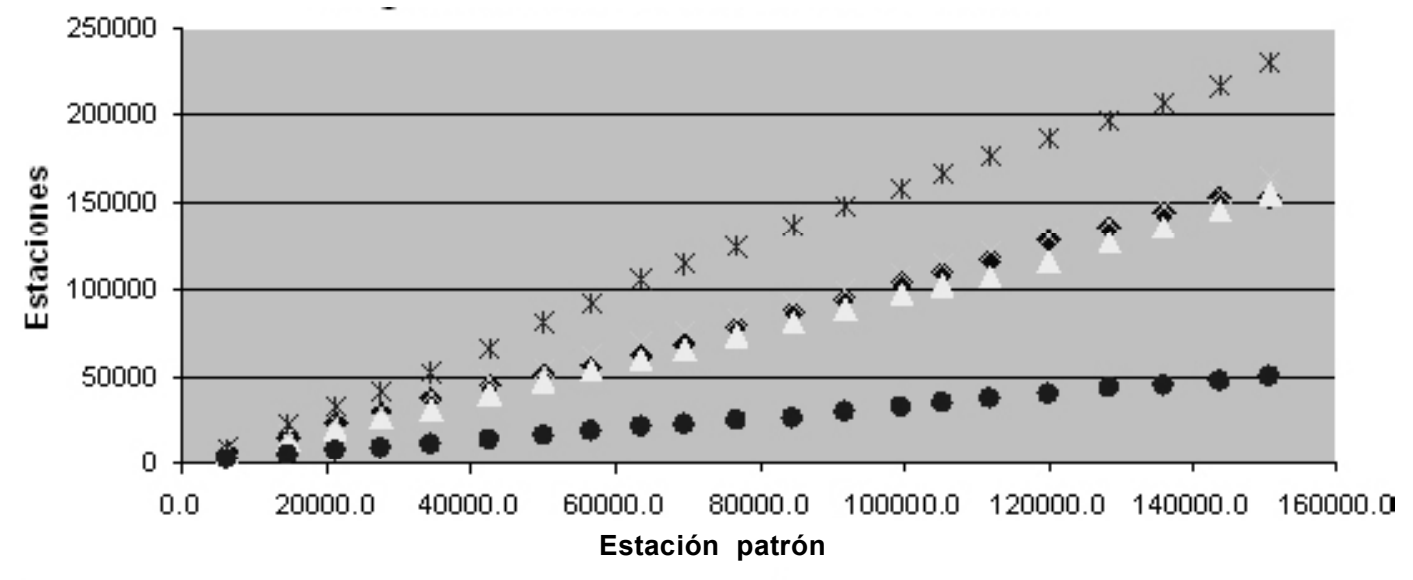

- Lloró - San Nicolás El Piñol × Ap Quibdó * Tutunendo * Carmen de Atrato

Gráfica 1. Análisis de curva de doble masa, para las 7 estaciones escogidas (precipitación anual acumulada 1983-2003)

Tratamiento de datos. Es frecuente encontrar datos faltantes o erróneos en series climatológicas «especialmente en las series de precipitación», debido a que toda medición se ve afectada por algún tipo de error (sistemático o accidental). De esta forma se escogió el período en el cual las estaciones presentaron menor cantidad de datos faltantes, siendo el período 1983 a 2003 (20 años) para el cual cumplieron 7 estaciones (Tabla 1).

Estimación de datos faltantes. En esta fase se llevó a cabo una revisión y verificación de los datos en cada estación, calculando con la ayuda de los programas Star Graphy y Excel los parámetros estadísticos básicos como media, máximo, mínimo, desviación estándar, coeficiente de variación, gráficos de dispersión y varianza; De esta manera se descartaron los datos erróneos y posteriormente se realizó la estimación y complementación de los datos faltantes en las series, utilizando el paquete estadístico TRAMO (Guerrero \& Nieto, 1999), que utiliza modelos arima (modelos autoregresivos integrados de medias móviles) para el relleno de datos faltantes y la realización de pronósticos. A través del procedimiento anterior se organizaron series completas mensuales de la variable precipitación para el período 1983-2003 en las 7 estaciones mencionadas arriba.

Análisis de la consistencia de las series. Para la evaluación espacial de la precipitación en el área de estudio se verificó la consistencia y la homogeneidad de las series durante el período escogido (1983-2003), es decir, que las estaciones hayan permanecido durante el período de observaciones en las mismas condiciones sin cambiar el instrumental o que las condiciones del predio no hayan sido alteradas significativamente. Para detectar dicha homogeneidad y consistencia se empleó el método de curvas de doble masa (MDM)(Gráfica 1) o doble acumulada(CDA) (Thomas B. 1963 y Donald Gabriels et al., 2004) y la prueba de Rachas (H.C. Thom, 1966) para cada estación, garantizando así la calidad de la información. 
Balance hídrico de la cuenca alta del Atrato. W Murillo, et al.

Tabla 2

Precipitación media mensual en mm, durante el período 1983-2003, para las estaciones de la cuenca alta del río Atrato

\begin{tabular}{lcccccccccccc}
\hline Estación & Ene & Feb & Mar & Abr & May & Jun & Jul & Ago & Sep & Oct & Nov & Dic \\
\hline Bagadó & 530.6 & 401.8 & 485.8 & 614.3 & 781.6 & 680.8 & 620.8 & 644.4 & 684.5 & 755.0 & 806.0 & 636.8 \\
Lloró & 566.9 & 489.4 & 494.5 & 715.6 & 741.8 & 703.3 & 696.5 & 732.1 & 741.5 & 606.1 & 696.2 & 555.2 \\
San Nicolás & 106.9 & 115.0 & 130.7 & 185.4 & 244.9 & 214.1 & 246.3 & 184.4 & 210.6 & 238.9 & 216.6 & 130.4 \\
El Piñol & 514.2 & 362.2 & 467.2 & 589.9 & 800.9 & 669.2 & 561.3 & 568.8 & 658.6 & 783.2 & 850.5 & 638.4 \\
Ap Quibdó & 505.9 & 458.6 & 498.2 & 690.8 & 736.5 & 704.3 & 814.0 & 826.7 & 701.0 & 598.1 & 697.8 & 584.3 \\
Tutunendo & 811.9 & 628.3 & 579.5 & 843.9 & 975.3 & 959.1 & 1074.3 & 1082.1 & 1137.9 & 1018.0 & 994.3 & 826.0 \\
Carmen deAtrato & 116.1 & 123.6 & 140.2 & 198.0 & 260.0 & 228.0 & 261.7 & 198.6 & 224.8 & 253.4 & 230.6 & 141.0 \\
\hline
\end{tabular}

Vale mencionar que después del tratamiento y análisis los datos ninguna de las series de la cuenca alta del río Atrato presentaron heterogeneidad.

\section{ANÁLISIS Y DISCUSIÓN}

Comportamiento de la precipitación en la cuenca alta del río Atrato. La Tabla 2 y la Figura 3, muestran el comportamiento de la precipitación acumulada obtenida por los valores medios mensuales para la cuenca alta del río Atrato durante el período 1983-2003, para cada una de las estaciones dentro del área de estudio cuyo orden se encuentra denotado de la siguiente forma: 1. Bagadó, 2. Lloró, 3. San Nicolás, 4. El Piñol, 5. AP Quibdó, 6. Tutunendo, 7. Carmen de Atrato. La cuenca alta del río Atrato en general presenta una precipitación media de $6711.7 \mathrm{~mm}$ (media aritmética de todas las estaciones).

Como se puede apreciar la precipitación media mensual en todas las estaciones presenta un comportamiento de tipo bimodal durante el año, siendo menos marcados en Lloró, Apto Quibdó y Tutunendo (zona baja). Además su primer máximo se encuentra entre los meses de abril y mayo, y su primer mínimo entre los meses de enero y febrero, siendo febrero el de menor valor para todas, a excepción de Tutunendo en abril. El segundo máximo se encuentras entre los meses de julio y agosto, agosto para las estaciones de AP Quibdó, Tutunendo y Lloró, en cambio para el resto de estaciones este comienza entre los meses de septiembre y octubre.

El comportamiento de tipo bimodal que se registra en la cuenca es asociado al doble desplazamiento latitudinal de la zona de confluencia intertropical sobre Colombia (Eslava 1994).

Las mayores precipitaciones que se registran en la cuenca se presentan al occidente y a una altitud menor de 800 metros (en la zona baja), mientras que al oriente de la cuenca (parte alta) el régimen de precipitación decrece considerablemente con la altura hasta en $72 \%$ en comparación con la zona baja. Las mayores precipitaciones anuales en la zona de estudio son registradas en la estación de Tutunendo con valores de 10930,6 mm, seguidos de los de la estación Apto de Quibdó con valores de 7816,2 $\mathrm{mm}$ y las menores cantidades de precipitación anual se presentan en la estación del Carmen del Atrato con valores de $2376,1 \mathrm{~mm}$.

En la zona occidental de la cuenca (Figura 5) las dos temporadas lluviosas que se presentan, demuestran porcentualmente diferencias significativas que hay que tener en cuenta. En la primera temporada lluviosa hay cerca de 60 $\%$ de la precipitación total anual; en cambio en la segunda temporada lluviosas sólo se presenta 10\% (transición); en la temporada menos lluviosa (enero-marzo) sólo se presenta $20 \%$ de la precipitación total anual y en la segunda temporada que cubre sólo un mes (octubre) se presenta $9 \%$ del total anual; este mes se puede considerar como una temporada de transición entre dos períodos más lluvioso para la zona occidental de la cuenca (Gráfica 2).

Con respecto a la zona oriental, la primera temporada lluviosa solo representa $40 \%$ de la precipitación total anual; en cambio en la segunda temporada lluviosa sólo se presenta $30 \%$; en la temporada de menos lluvia (enero-marzo) sólo se presenta $16 \%$ de la precipitación total anual, y en la segunda temporada que cubre (agosto septiembre) $18 \%$ del total anual (Gráfica 3).

En promedio, para las estaciones del occidente de la cuenca, los valores máximos no superan los $1137.9 \mathrm{~mm}$ de lluvia y los valores mínimos no bajan de los 362 .mm; en cambio para la zona oriente los valores máximos no superan los 260 $\mathrm{mm}$, y los mínimos no bajan de $106 \mathrm{~mm}$ (Tabla 2).

Estimación de la precipitación media por isoyetas. En esta técnica se realizó un mapa de isoyetas (líneas trazadas en un plano que representan puntos de igual precipitación) para 
Bioetnia Volumen 5 № 2 (julio-diciembre), 2008

6

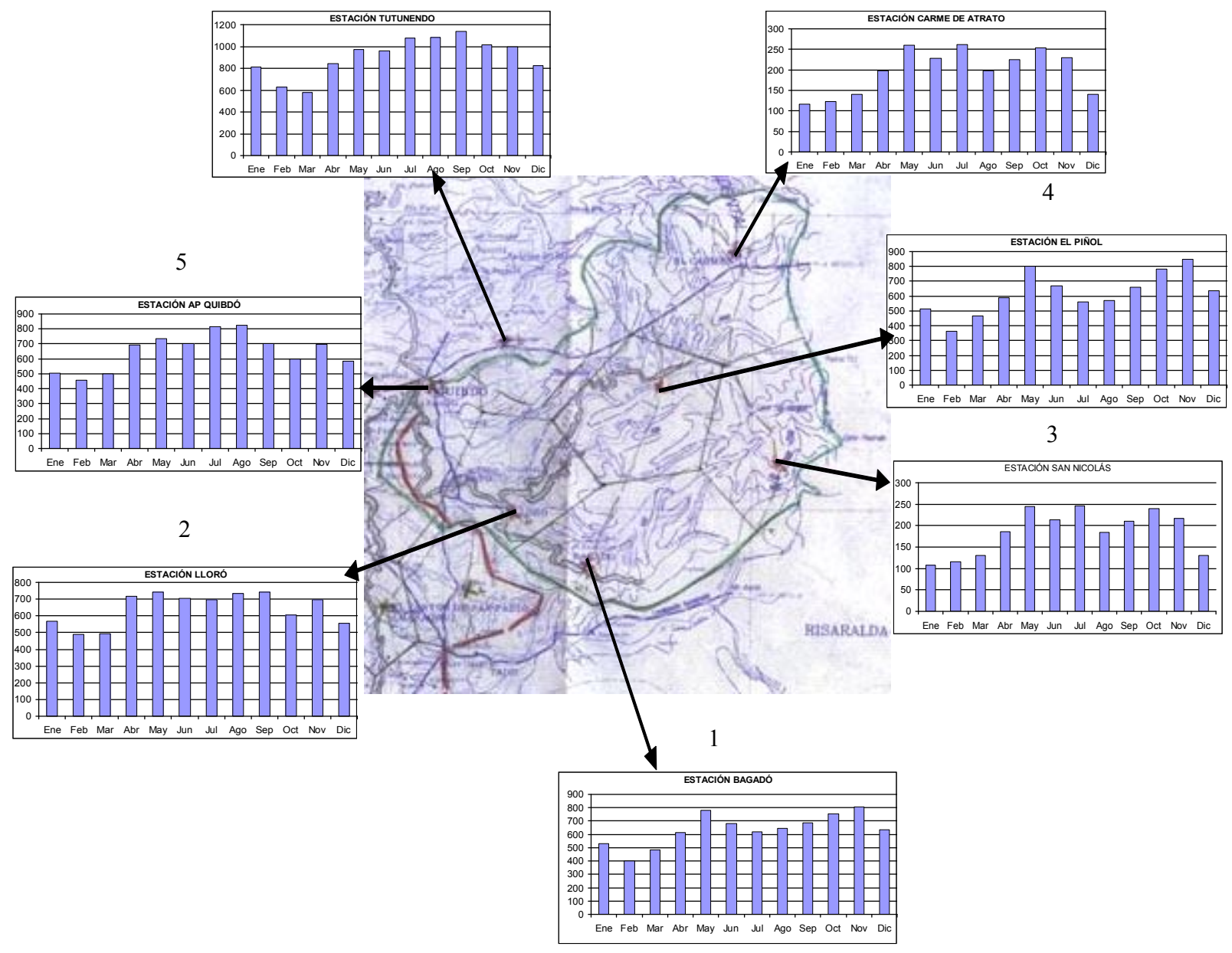

Figura 3. Distribución espacial de la precipitación media mensual en la cuenca del río Quito, durante el período 1983-2003. 1. Bagadó, 2. Lloró, 3. San Nicolás, 4. El Piñol, 5. AP Quibdó, 6. Tutunendo, 7. Carmen de Atrato.

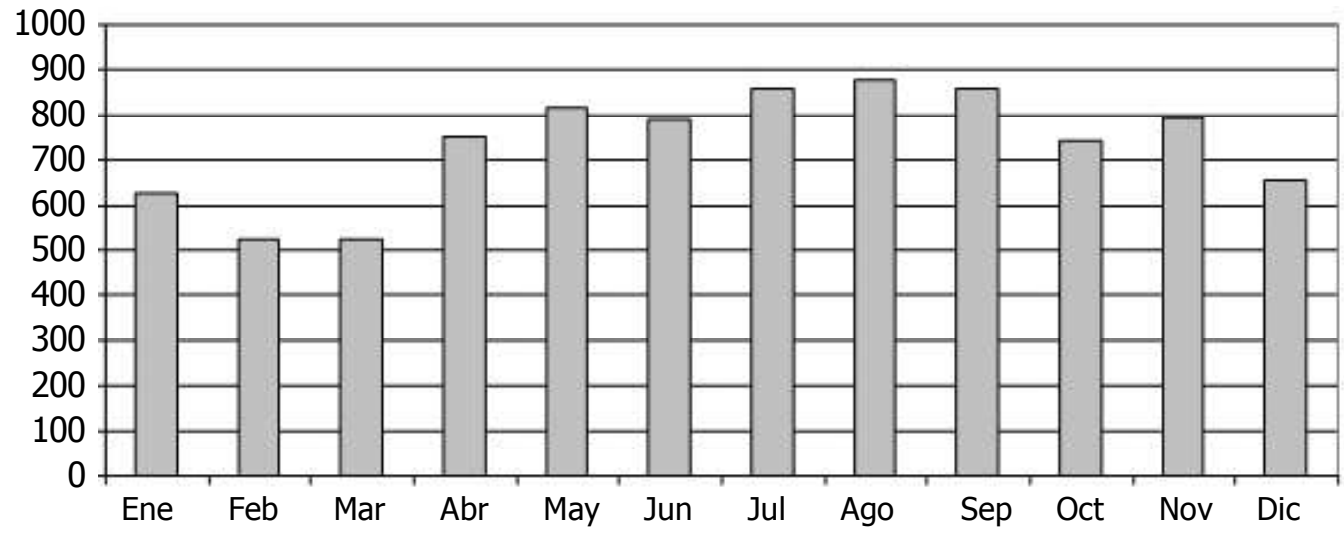

Gráfica 2. Valores medios mensuales de precipitación acumulada en la zona alta de la cuenca alta del río Atrato años 1983-2003. 
Balance hídrico de la cuenca alta del Atrato. W Murillo, et al.

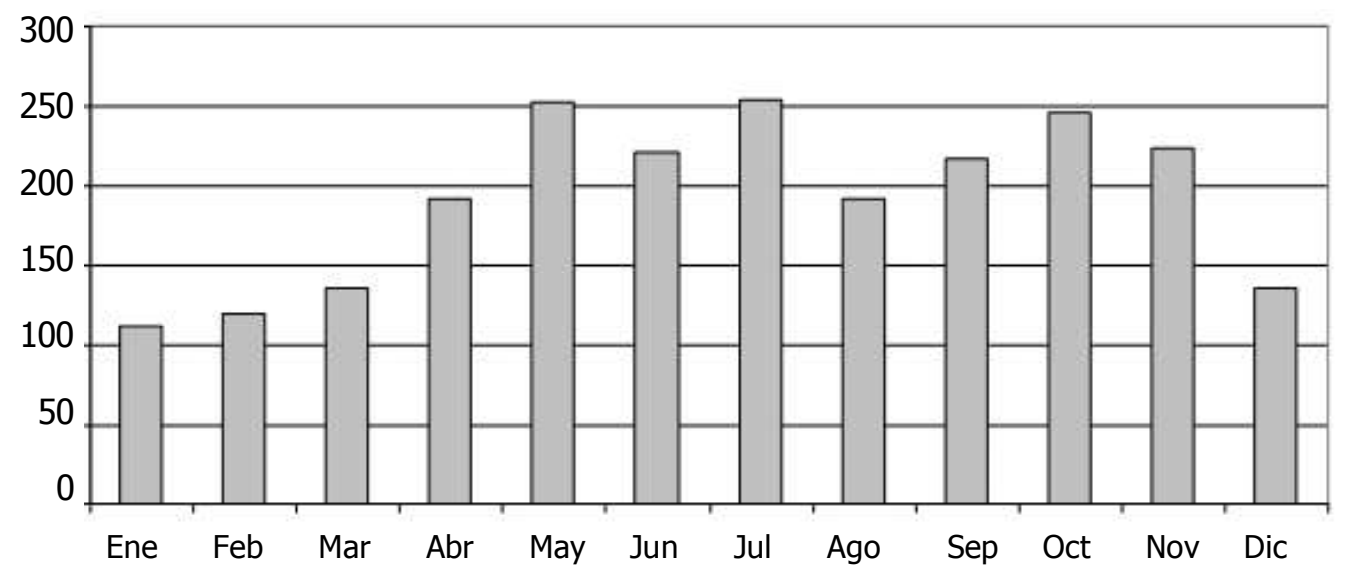

\section{Gráfica 3. Valores medios mensuales de la precipitación acumulada en zona alta de la cuenca alta del río Atrato años 1983-2003}

la cuenca en estudio considerando los datos de precipitación media de cada una de las estaciones(Figura 4). Al aplicar esta técnica es necesario calcular el área de la cuenca para la cual se utilizó un planímetro digital marca Planix 5 «Tamaya» con una resolución de $0.1 \mathrm{~cm}$ cuadrados y una precisión de $\pm 0.2 \%$, obteniendo los valores expuestos en la Tabla 3.

Aplicando la ecuación (1) para las áreas y las precipitaciones medias resultantes del método de isoyetas (Tabla 3), se obtiene una precipitación media para la cuenca alta del río Atrato de $58777 \mathrm{~mm}$

$$
P m=\frac{\sum_{i=1}^{n}\left[\frac{(P i+1+P i)}{2}\right] * S_{i+1, i}}{S t}
$$

Ecuación (1)

De donde:

$\mathrm{Pm}_{\mathrm{j}}=$ Precipitación media del área geográfica en estudio, en el tiempo $\mathrm{j}(\mathrm{mm})$.

$\mathrm{P}_{\mathrm{i}+1}=$ Precipitación de la isoyeta $\mathrm{i}+1(\mathrm{~mm})$.

$\mathrm{P}_{\mathrm{i}}^{\mathrm{i}+1}=$ Precipitación de la isoyeta $\mathrm{i}$

$\mathrm{S}_{\mathrm{i}+1, \mathrm{i}}=$ Superficie de influencia por dos isoyetas secuenciales i $\left(\mathrm{km}^{2}\right)$.

$P_{\mathrm{ij}}=$ Precipitación de la estación $\mathrm{i}$, en el tiempo j $(\mathrm{mm})$.

$\mathrm{S}_{\mathrm{t}}=$ Superficie total del área de estudio $\left(\mathrm{km}^{2}\right)$

La Tabla 3 presenta los datos de las 10 áreas resultantes del método de isoyetas, siendo la zona A1 el área de mayor representatividad $\left(1172.5 \mathrm{~km}^{2}\right)$ y la región A7 el área de menor representatividad con $40 \mathrm{~km}^{2}$. En cuanto a las precipitaciones medias resultantes, la zona A11 es la de mayor cantidad de precipitación media con $9500 \mathrm{~mm}$ de lluvia, siendo además la de mayor intensidad, y la región A7 y A9 las que presenta menor cantidad de precipitación con $1500 \mathrm{~mm}$ de lluvia. 


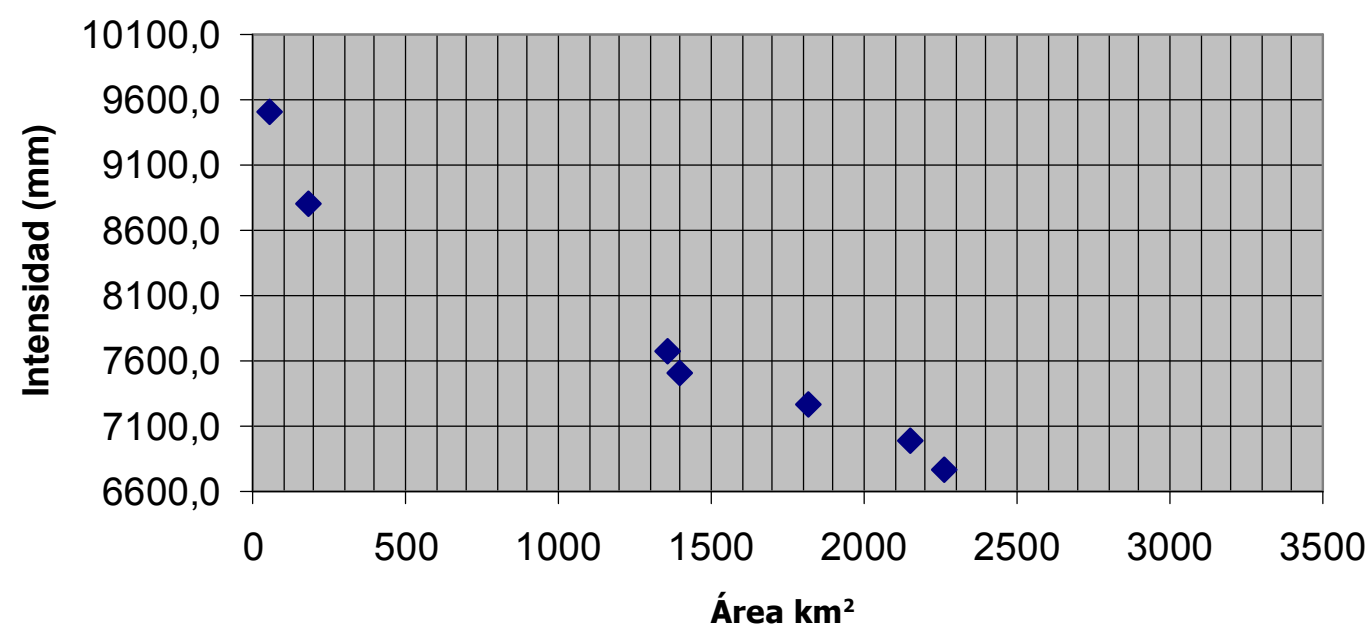

Gráfica 4. Curva de intensidad área para los resultados del método de isoyetas en la cuenca alta del río Atrato (1977-2001)
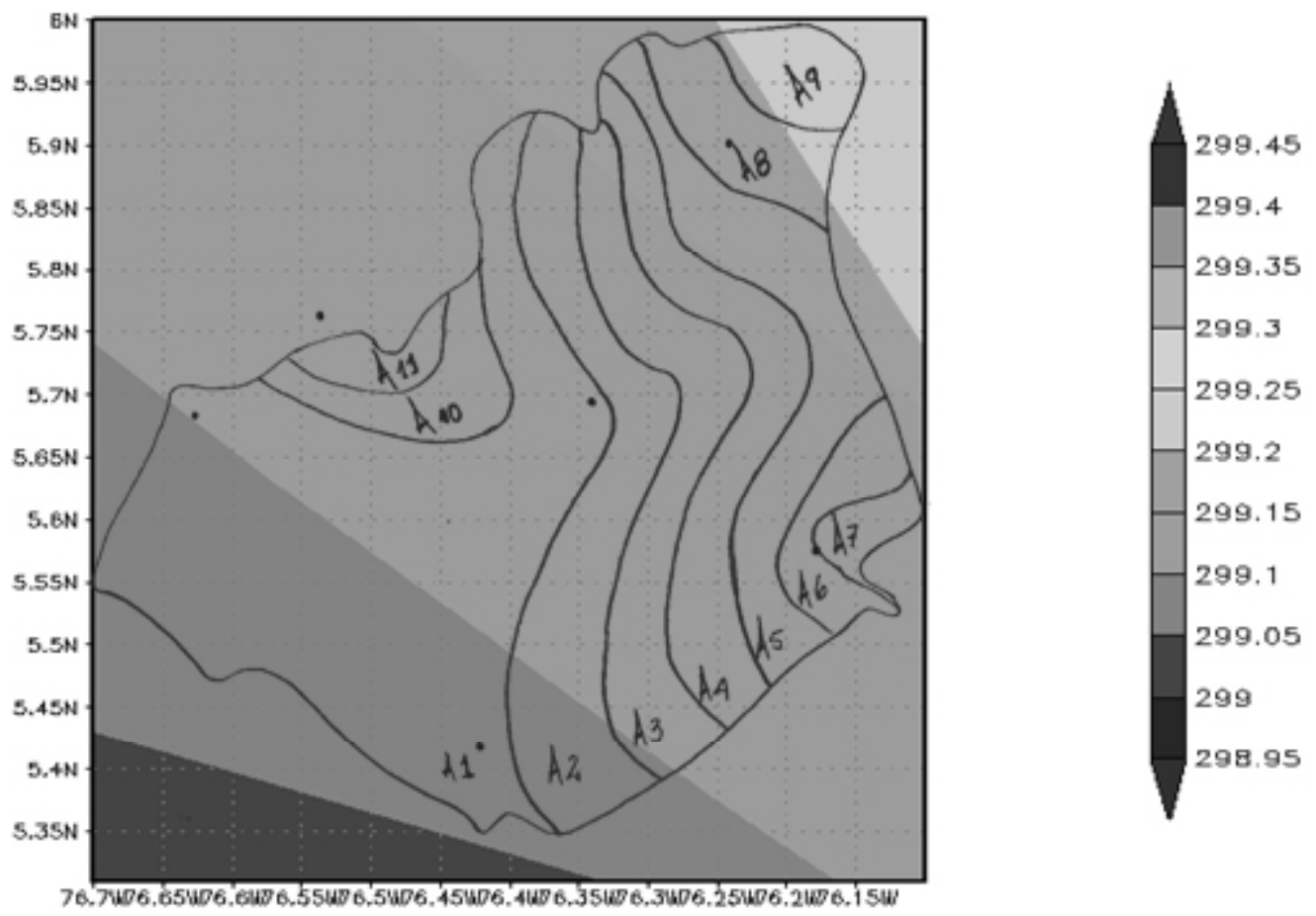

Figura 5. Temperatura media $\left({ }^{\circ} \mathrm{C}\right)$ para el área de estudio en contorno de grises tomada del reanálisis, período 1967-2001. Precipitación a través del método de isoyetas

- Perímetro: la cuenca alta del río Atrato obtuvo un perímetro de $208,5 \mathrm{~km}$.

- Superficie: utilizando el planímetro digital descrito con anterioridad se obtuvo un valor de la superficie de la cuenca de $3118,75 \mathrm{~km}^{2}$

- Longitud del río principal: el rió principal (rió Atrato) obtuvo una longitud de $109 \mathrm{~km}$.

- Coeficiente de compacidad (Cc): se obtiene a través de la ecuación(2), (si el resultado es 1 la cuenca es circulary entre más se aleje de 1 la cuenca es más alongada), obteniendo un valor de 1,43 (cuenca alongada).

Ecuación(2) $\mathrm{Cc}=0.28 \times \frac{P}{\sqrt{A}}$

De donde:

$\mathrm{P}=$ perímetro de la cuenca 
Tabla 3

Precipitación media y áreas obtenidas a través de la técnica de isoyetas

\begin{tabular}{|c|c|c|}
\hline No & $\begin{array}{l}\text { Método de isoyetas } \\
\text { Precipitación media }(\mathrm{mm})\end{array}$ & Área $\mathbf{k m}^{2}$ \\
\hline $\mathrm{A} 1$ & 7500 & 1172,5 \\
\hline A2 & 6500 & 420 \\
\hline A3 & 5500 & 332,5 \\
\hline A4 & 4500 & 307,5 \\
\hline A5 & 3500 & 315 \\
\hline A6 & 2500 & 112,5 \\
\hline A7 & 1500 & 40 \\
\hline A8 & 2500 & 140 \\
\hline A9 & 1500 & 93,75 \\
\hline A10 & 8500 & 130 \\
\hline A11 & 9500 & 55 \\
\hline Totales & 5877,7 & 3118,75 \\
\hline
\end{tabular}

$\mathrm{A}=$ área o de la cuenca

- Factor de forma $(F f)$ : este se obtiene por la relación entre la superficie de la cuenca y el cuadrado de la longitud del río principal (si el resultado es 1 la cuenca es cuadrada), dando un valor de 0,26 indicativo de una forma ligeramente alargada.

- Densidad de drenaje (Dd): es la relación entre la longitud de todos los ríos de la cuenca y el área de la cuenca. Para el cálculo de esta característica se calculó la longitud de todos los ríos y quebradas dentro de la cuenca (Tabla 4 ) y se obtuvo un valor de $\mathrm{Dd}=0,11$. Este resultado indica la presencia de longitudes de 110 metros de corrientes de agua por cada kilómetro cuadrado.

- Extensión media de escurrimiento superficial (Dm): se obtiene a través de la ecuación (3), con un valor de 2,24

$$
\text { Ecuación(3) } \quad D m=\frac{A}{4 \cdot L r}
$$

De donde:

$\mathrm{A}=$ área de la cuenca

$\mathrm{Lr}=$ longitud de todos los ríos en la cuenca

Para obtener el cálculo del $D m$ fue necesario medir la longitud de los ríos y quebradas existentes en la cuenca (Tabla4).

En la cuenca alta del río Atrato se encuentran ríos de gran longitud como lo son Andagueda, Taro y Mumbaradó con 76, 35 y $32.5 \mathrm{~km}$ respectivamente; en total se localizan sobre el área de estudio 16 ríos relacionados con el río principal.

- Pendiente del río principal. El río principal arrojó una pendiente ligera aproximada al 5.8\% empleando la función
Tabla 4

Longitud de ríos y quebradas pertenecientes a la cuenca alta del río Atrato

\begin{tabular}{|lc}
\hline Nombre del río o quebrada & Longitud $(\mathbf{k m})$ \\
\hline Río Andagueda & 76,0 \\
Río Capa & 15,5 \\
\hline Río Salao & 9,5 \\
Río Churina & 12,5 \\
\hline Río Yuto & 17,5 \\
Río Tumutumbudo & 13,5 \\
\hline Río Tanando & 26,0 \\
Río Purre & 17,0 \\
\hline Río Murumbi & 23,5 \\
Río Mumbaradó & 32,5 \\
\hline Río Diamante & 17,0 \\
Río Cabí & 20,5 \\
\hline Río Giraldot & 12,5 \\
Río Palestina & 8,0 \\
\hline Río Gicaradocito & 11,0 \\
Río Taro & 35,0 \\
\hline Total & 347,5 \\
\hline
\end{tabular}

tangente para la elevación del río y la distancia horizontal desde su nacimiento hasta la desembocadura, con la ayuda de las cartografías de la zona.

- Pendiente de la cuenca. Se calculó a través de la ecuación (4), teniendo en cuenta mapas cartográficos del área de estudio que presentaban la distancia entre curvas de nivel cada 25 metros. Se obtuvo un resultado de $18 \%$ siendo una pendiente mayor que la que presenta el río principal.

Ecuación(4) $\mathrm{P}=1.5 \times \frac{D \cdot n}{L}$

De donde:

$\mathrm{P}=$ pendiente de la cuenca

$\mathrm{D}=$ distancia entre curvas de nivel

$\mathrm{n}=$ número de cortes en los ejes elegidos por las curvas de nivel

$\mathrm{L}=$ longitud de los ejes elegidos

Cálculo de la escorrentía en la cuenca. Se fundamentó en el balance estimado a partir de las variables precipitación y evapotranspiración.

Cálculo de la evapotranspiración. Para el cálculo de la evapotranspiración potencial en la cuenca alta del río Atrato, se utilizo la ecuación (5) propuesta por TuKe (1978), para la cual se emplean datos de temperatura media. Debido a la falta 
Tabla 5

\section{Evapotranspiración, para la cuenca alta del río Atrato utilizando la ecuación propuesta por Tuke(1978)}

\begin{tabular}{lccc}
\hline $\begin{array}{l}\text { Área } \\
\text { No }\end{array}$ & $\begin{array}{c}\text { Precipitación } \\
\text { media }(\mathbf{m m})\end{array}$ & $\begin{array}{c}\text { Temperatura } \\
\text { media }\left({ }^{\circ} \mathbf{C}\right)\end{array}$ & $\begin{array}{c}\text { Evapotranspi- } \\
\text { ración ETR }\end{array}$ \\
\hline A1 & 7500 & 26,05 & 42,8 \\
\hline A1' & 7500 & 26,15 & 43,0 \\
\hline A2 & 6500 & 26,05 & 42,8 \\
\hline A2' & 6500 & 26,15 & 43,0 \\
A3 & 5500 & 26,15 & 43,0 \\
\hline A4 & 4500 & 26,15 & 43,0 \\
A4' & 4500 & 26,25 & 43,1 \\
\hline A5 & 3500 & 26,15 & 43,0 \\
\hline A5' & 3500 & 26,25 & 43,1 \\
\hline A6 & 2500 & 26,15 & 43,0 \\
\hline A7 & 1500 & 26,15 & 43,0 \\
A8 & 2500 & 26,25 & 43,1 \\
\hline A8' & 2500 & 26,30 & 43,2 \\
A9 & 1500 & 26,30 & 43,2 \\
\hline A10 & 8500 & 26,15 & 43,0 \\
\hline A11 & 9500 & 26,15 & 43,0 \\
\hline
\end{tabular}

de información sobre la temperatura del aire en la zona, se utilizaron los datos del reanálisis (NCEP/NCAR) los cuales presentan un período mayor a 30 años para esta variable en los puntos de grilla correspondientes al área de estudio (Figura 5).

Ecuación(5) $\quad E T r=\frac{\mathrm{P}}{\sqrt{0.9+\mathrm{P}^{2} / L_{2}}}$

De donde:

$\mathrm{P}=$ Precipitación media

$\mathrm{L}_{2}=300+25 \mathrm{~T}+0.05 \mathrm{~T}^{3}$.

En donde, $\mathrm{T}$ es la temperatura media.

La Figura 5 presenta en contornos, los valores de la temperatura media (en grados Kelvin) de modo espacial sobre la cuenca alta del río Atrato, para el período 1967-2004. Además, presenta el trazado de isoyetas brindando así la información de la precipitación. Se observa cómo la temperatura media del aire en el área de estudio oscila entre $\operatorname{los} 26.05^{\circ} \mathrm{C}$ (contorno claro) y los $26^{\circ} .3$ (contorno oscuro), siendo muy pequeña la variación en la cuenca.

Las áreas A3, A6, A7, A10 y A11 quedaron ubicadas en zonas más claras de la Figura 5, es decir, que presentan una
Tabla 6

Valores de escorrentía para cada una de las áreas en la cuenca alta del río Atrato

\begin{tabular}{lccc}
\hline $\begin{array}{l}\text { Área } \\
\text { No }\end{array}$ & $\begin{array}{c}\text { Precipitación } \\
\text { media (mm) }\end{array}$ & $\begin{array}{c}\text { Temperatura } \\
\text { media }\left({ }^{\circ} \mathbf{C}\right)\end{array}$ & $\begin{array}{c}\text { Evapotranspi- } \\
\text { ración ETR }\end{array}$ \\
\hline A1 & 7500 & 42,8 & 7457,2 \\
\hline A1' & 7500 & 43,0 & 7457,0 \\
\hline A2 & 6500 & 42,8 & 6457,2 \\
\hline A2' & 6500 & 43,0 & 6457,0 \\
\hline A3 & 5500 & 43,0 & 5457,0 \\
\hline A4 & 4500 & 43,0 & 4457,0 \\
\hline A4' & 4500 & 43,1 & 4456,9 \\
\hline A5 & 3500 & 43,0 & 3457,0 \\
\hline A5' & 3500 & 43,1 & 3456,9 \\
\hline A6 & 2500 & 43,0 & 2457,0 \\
\hline A7 & 1500 & 43,0 & 1457,0 \\
\hline A8 & 2500 & 43,1 & 2456,9 \\
\hline A8 & 2500 & 43,2 & 2456,8 \\
\hline A9 & 1500 & 43,2 & 1456,8 \\
A10 & 8500 & 43,0 & 8457,0 \\
\hline A11 & 9500 & 43,0 & 9457,0 \\
\hline
\end{tabular}

temperatura media de $26.15^{\circ} \mathrm{C}$ y el área $\mathrm{A} 9$ mostró valores de $26.3^{\circ} \mathrm{C}$. Las áreas A 1 y A2 con valores de $26.05^{\circ} \mathrm{C}$ y $26.15^{\circ} \mathrm{C}$, debido a esto las áreas A1 y A2 se dividieron de la siguiente manera: A1 $=$ A1 contorno oscuro y A1' contorno semioscuro; $\mathbf{A 2}=$ A2 contorno semi-oscuro y A2' contorno semiclaro.

Las áreas A4 y A5 se encuentran dentro de la zona que presenta valores de $26.15^{\circ}$ y de $26.25^{\circ} \mathrm{C}$, y se dividieron de la siguiente manera: $\mathbf{A 4}=\mathrm{A} 4$ contorno semi-claro y A4' contorno semi-claro; $\mathbf{A 5}=\mathrm{A} 5$ contorno semi- claro y A5' contorno semi-claro.

Por último el área $\mathrm{A} 8$ se le aplicó el mismo proceso de división que las anteriores quedando así: $\mathbf{A 8}=\mathrm{A} 8$ contorno semi-claro y A8' contorno claro.

Aplicando la ecuación (5) en cada área (A) se obtiene el valor correspondiente de evapotranspiración a nivel espacial en el área de estudio (Tabla 5). Aplicando la ecuación (5) en cada área (A) se obtiene el valor correspondiente de evapotranspiración a nivel espacial en el área de estudio (Tabla 6).

Los mayores valores de evapotranspiración se presentaron en las áreas A8, A9, A 10 y A1 1 con un registro de 43 mm; el resto obtuvieron un valor de $42 \mathrm{~mm}$.

La variación de la evapotranspiración en la cuenca de estudio no es muy significativa, debido a que los valores obtenidos para la temperatura del aire a través de los datos del 
reanálisis no presentan variaciones significativas.

Cálculo de la escorrentía en la cuenca. Debido a la carencia de datos hidrometeorológicos para el área de estudio que permitieran realizar un óptimo balance hídrico, se optó por utilizar la información existente (precipitación, evapotranspiración y temperatura) aplicando la ecuación (5), calculando la escorrentía (Q) para la cuenca alta del río Atrato (Tabla 6).

Ecuación(5) $\quad \mathrm{Q}=\mathrm{P}-\mathrm{ETR}$

De donde:

$\mathrm{Q}=$ escorrentía

$\mathrm{P}=$ precipitación media

$\mathrm{ETR}=$ evapotranspiración potencial

La escorrentía total presenta los mayores valores en las áreas A1 A10 y A11 y los menores valores en las áreas A6, A7 y A8 (Figura 5).

\section{CONCLUSIONES}

Se comprueba que la variación del ciclo anual de la precipitación describe una distribución de tipo bimodal con dos períodos de valores máximos y dos mínimo, siendo uno de estos mínimos más bien un mes de transición. En cambio para las estaciones de la zona oeste o parte alta encontramos dos períodos máximo y dos períodos mínimos muy bien definidos. Se encontró que las mayores precipitaciones que se generan en la cuenca, se presentan en tal zona baja hasta en $75 \%$ en promedio, mientras que la zona alta recibe sólo $25 \%$ del promedio total.

Las mayores cantidades de precipitación se presentan en los meses de agosto, junio y julio, y septiembre en la zona baja, mientras que en la zona alta en los meses de mayo a julio en promedio. Con respecto a los valores mínimos, ambos reciben las menores cantidades en el mes de febrero y marzo, siendo febrero el mes con menor cantidad de precipitación sobre la cuenca y el segundo valor mínimo se presenta en el mes de octubre en la zona baja y en agosto en la zona alta, siendo un período de transición para ambas zonas con respecto a la segunda temporada de mayor cantidad de precipitación.

Se encontró que la estación de Tutunendo fue la que presentó los mayores valores de precipitación seguido de la estación AP Quibdó, y las menores en el Carmen de Atrato. La precipitación media en la cuenca alta del río Atrato, presenta un valor de 5877,7 $\mathrm{mm}$ de lluvia (método de isoyetas). Las diferencias entre las precipitaciones medias calculadas a través de los distintos métodos no son significativas, exceptuando el de la media aritmética, que es el más desfasado con una diferencia de $830 \mathrm{~mm}$. En cambio con los otros métodos no supera los $151 \mathrm{~mm}$.
En el cálculo de la precipitación a través del método de isoyetas se obtiene un valor medio de $5877,7 \mathrm{~mm}$, y un total acumulado $728250,0 \mathrm{~mm}$. Se presentóun gran núcleo de concentración de precipitación hacia el oeste de la cuenca con un valor de $9500 \mathrm{~mm}$, y dos núcleos mínimos hacia el este con valores de $2000 \mathrm{~mm}$. La escorrentía en la cuenca es de $4820 \mathrm{~mm}$, presentando los mayores valores hacia el oeste. La evapotranspiración potencial en el área de estudio presenta un promedio de $42 \mathrm{~mm}$.

Las curvas de intensidad y duración en elárea de la cuenca se presentaron entre los 50 y $100 \mathrm{~km}^{2}$ con intensidades de lluvias por encima de los $8.700 \mathrm{~mm}$. La cuenca alta del río Atrato mostró las siguientes características: en un área de $3118,9 \mathrm{~km}^{2}$, con un perímetro de $285 \mathrm{~km}$, la longitud del río principal (río Atrato) es de $109 \mathrm{Km}$, con un coeficiente de capacidad de 1,43 (cuenca alongada), dando un factor de forma 0,26 indicativo de una forma ligeramente alargada y una densidad de drenaje de 0.11 ; en la extensión del escurrimiento superficial se encontró que ríos de gran longitud como Andagueda, Taro y Mumbaradó tienen valores de 76, 35 y 32.5 km respectivamente.

La pendiente de la cuenca del río Atrato obtuvo un valor de $2.5^{\circ}$ y la pendiente del río principal $3.6^{\circ}$, siendo ésta pendiente mayor en comparación con la pendiente de la cuenca. En cambio el río principal arrojó una pendiente ligera aproximada $5.8 \%$. Los mayores valores de evapotranspiración se presentaron en las áreas A8, A9, A10 y A11 con un registro de $43 \mathrm{~mm}$, el resto obtuvo un valor de $42 \mathrm{~mm}$.

\section{LITERATURA CITADA}

Eslava, J.A. 1994. Climatología del Pacífico colombiano. Colección Eratóstenes, $\mathrm{N}^{\mathrm{o}}$ 1. Santa Fe de Bogotá D.C.: Academia Colombiana de Ciencias Geofísicas. 79 p.

Gandin, L. 1970. The planning of Meteorological Station Networks, WMO Technical Note $\mathrm{N}^{\circ} 111$, IP. 149, Suiza.

Gentry, A. 1990. Selva húmeda tropical. Introducción. En: Villegas B. (ed.) Selva húmeda de Colombia. Bogotá: Villegas Editores. p. 13-52.

Guerrero, V., Nieto, F.H. 1999. Temporal and contemporaneous disaggregation of multiple time series. Test. 8 (2): 459-89.

Gabriels, D., Ovalles, F., Santibáñez, F., Moyano, M.C., Aguilera, R., Pizarro, R., et al. 2004. Guía metodológica para la elaboración del mapa de zonas áridas, semiáridas y subhúmedas secas de América Latina y el Caribe. Paris: CAZALAC ORG. p. 1-59.

Kalnay E., M. Kanamitsu, R. Kistler, W. Collins, D. Deaven, L. Gandin, et al. 1996. The NCEP/NCAR 40-year reanalysis project. Bull Am Meteorol Soc. 77: 437-71.

Ramírez, A y N. Bustos, 1977. Estudio preliminar de precipitación y caudales medios en la vertiente del Pacífico. Proyecto de grado. Bogotá: Fundación Universitaria «Jorge Tadeo Lozano», Facultad de Ingeniería Geológica.

Searcy, J. K., Hardison C. H. 1960. Double Mass Curves. U.S. Geological Survey, Water-Supply Paper 1541-B. p. 66.

Trojer, H. 1958. Meteorología et climatología de la vertiente del Pacífico colombiano. Rey Acad Colomh Ci Ex Fis Nat. 10 (40): 199-219.

Thom H.C. 1966. Some methods of climatological analysis. Tecnichal note $\mathrm{N}^{\mathrm{o}}$ 81. World Meteorologicat Organization. 52 p.

Tukey, J.W. 1977. Exploratory data analysis. Addison-Wesley. 